\title{
Image in Painting Techniques: A survey
}

\author{
Komal s Mahajan ${ }^{1}$, Prof. M. B. Vaidya ${ }^{2}$ \\ ${ }^{1,2}$ (Computer Engineering Department, AVCOE, Ahamadnagar, India)
}

\begin{abstract}
Inpainting is the process of reconstructing lost or deteriorated part of images based on the background information. i. e. image Inpainting fills the missing or damaged region in an image utilizing spatial information of its neighbouring region. Inpainting algorithm have numerous applications. It is helpfully used for restoration of old films and object removal in digital photographs. It is also applied to red-eye correction, super resolution, compression etc. The main goal of the Inpainting algorithm is to modify the damaged region in an image in such a way that the inpainted region is undetectable to the ordinary observers who are not familiar with the original image. There have been several approaches proposed for the image inpainting. This proposed work presents a brief survey of different image inpainting techniques and comparative study of these techniques. In this paper we provide a review of different techniques used for image Inpainting. We discuss different inpainting techniques like image PDE based image inpainting, Exemplar based image inpainting, hybrid inpainting, and texture synthesis based image inpainting and semi-automatic and fast digital Inpainting.
\end{abstract}

Keywords: Exemplar, Eye-correction, Hybrid Inpainting, Inpainting, Object Removal, Patch Propagation, Texture Synthesis,

\section{INTRODUCTION}

Nowadays, the image Inpainting technology is a hotspot in computer graphics. And it has important value in a heritage preservation, film and television special effects production, removing redundant objects etc. In the fine art museums, this Inpainting concept is used for degraded paintings. Conventionally Inpainting is carried out by professional artist and usually its very time consuming process because it was the manual process. The main goal of this process is to reconstruct damaged parts or missing parts of image. And this process reconstructs image in such a way that the inpainted region cannot be detected by a casual observer. Inpainting technique has found widespread use in many applications such as restoration of old films, object removal in digital photos, red eye correction, super resolution, compression, image coding and transmission. Image Inpainting reconstruct the damaged region or missing parts in an image utilizing spatial information of neighbouring region. Image Inpainting could also be called as modification and manipulation of an image. In image inpainting we would like to create original image but this is completely unfeasible without the prior knowledge about the image. In case of digital images we only have the image we are working on available to us and thus we are filling in a hole that encompasses an entire object. It is impossible to replace that entire object based on the present's information. Considering this as the aim of the inpainting algorithm is not only reconstruct what used to be in that hole. But instead to create a visually pleasing continuation of the data around the hole in such a way that it is not detectable by ordinary observer.

Diffusion based Inpainting was the first digital Inpainting approach. In this approach missing region is filled by diffusing the image information from the known region into the missing region at the pixel level. Basically these algorithms are based on theory of variational method and Partial Differential equation (PDE). The diffusion- based Inpainting algorithm produces superb results or filling the non-textured or relatively smaller missing region. The drawback of the diffusion process is it introduces some blur, which becomes noticeable when filling larger regions. All the PDE based in painting models are more suitable for completing small, non-textured target region.

The second category of Inpainting is exemplar- based Inpainting algorithm. This method of image Inpainting is an efficient approach to reconstructing large target regions. Exemplar-based Inpainting approach iteratively synthesizes the target region by most similar patch in the source region. These algorithms also overcome the drawbacks of PDE based inpainting. Also it removes smooth effect of the diffusion based Inpainting algorithm.

Most Inpainting methods work as follows:-

In the first step of Inpainting method the user manually selects the portions of the image that will be restored. The image restoration is done automatically, by filling these regions in with new information coming from the surrounding pixels or from the whole image. The algorithms proposed for Inpainting use the information from surrounding portions of image to inpaint the selected region.

There are mainly three approaches for inpainting as follows:- 
1. The first approach deals with the restoration of films.

2. The second class of algorithm deals with disocclusions.

3. Third class of algorithm deals with restoration of textures in the image.

This paper is organized as follows. Section II describes the techniques for the image Inpainting which including exemplar based Inpainting, PDE based Inpainting, and Texture Synthesis based Inpainting, Hybrid Inpainting, and Semi-automatic Inpainting. Finally concluding remarks are given in section III.

\section{DIFFERENT APPROACHES OF IMAGE INPAINTING}

Nowadays, there are different approaches to image inpainting are available. And we can classify them into several categories as follows:-

1. Texture Synthesis based Inpainting.

2. PDE based Inpainting.

3. Exemplar based Inpainting.

4. Hybrid Inpainting.

5. Semi-automatic and Fast Inpainting.

In this section we can briefly explain the concepts, advantages, drawbacks of the image Inpainting techniques.

\subsection{Texture Synthesis Based Inpainting}

Texture synthesis based algorithms are one of the earliest methods of image Inpainting. And these algorithms are used to complete the missing regions using similar neighbourhoods of the damaged pixels. The texture synthesis algorithms synthesize the new image pixels from an initial seed. And then strives to preserve the local structure of the image. All the earlier Inpainting techniques utilized these methods to fill the missing region by sampling and copying pixels from the neighbouring area. For e. g, Markov Random Field (MRF) is used to model the local distribution of the pixel. And new texture is synthesized by querying existing texture and finding all similar neighbourhoods. Their differences exist mainly in how continuity is maintained between existing pixels and Inpainting hole.

These synthesis based techniques perform well only for a select set of images where completing the hole region with homogenous texture information would result in nature completion. Then later, this technique was extended to fast synthesizing algorithm. This technique works by stitching together small patches of existing images referred to as image quilting. Bergen and Heeger [5] developed a parametric texture synthesis algorithm which can synthesize a matching texture, given a target texture. This was done by matching first order statistics of a linear filter bank which roughly match to the texture discrimination capabilities of Human Visual System [HVS]. Recently, a fast multi-resolution based image completion based and on texture analysis and synthesis was introduced fang et al in [6]. In their method, the input image was analysed by a patch based method using principal component analysis (PCA) and a Vector Quantization (VQ) based techniques was used to speed up the matching process of the texture inside the hole region.

The main objective of texture synthesis based inpainting is to generate texture patterns, which is similar to a given sample pattern, in such a way that the reproduced texture retains the statistical properties of its roottexture. And it does not appear simply as a tiled rearrangement of the root-texture. Contrary to vibrational inpainting (PDE based inpainting), the texture synthesis based methods can fill large textured regions, but depends on user choices on sampling location and content. Texture synthesis approaches can be categorized into 3 categories: Statistical (parametric), pixel-based and patch-based (non-parametric). Statistical methods are more likely to succeed in reproducing stochastic / irregular textures, but usually fail to reproduce structured/regular textures. On the other hand, pixel-based methods "build" on the sample texture pixel-by-pixel instead of applying filters on it, and their final outputs are of better quality than those of statistical methods, but they usually fail to grow large structured textures. Finally, patch-based [8] methods "build" on a sample texture patch-by-patch as opposed to pixel-by-pixel, thus they yield faster and more plausible regular textures.

Recently, a study for patch-based texture synthesis algorithms has shown that "for handling special types of texture we have to develop the special purpose algorithms". Taking this aim and the variety of algorithms for texture synthesis into consideration, we can conclude that there is no universal texture synthesizer is present. Still it remains a goal to be desire. The texture synthesis based Inpainting perform well in approximating textures. These algorithms have difficulty in handling natural images as they are composed of structures in form of edges. Also they have complex interaction between structure and texture boundaries. In some cases, they also require the user to specify what texture to replace and the place to be replaced. Hence while appreciating the use of texture synthesis techniques in Inpainting, it is important to understand that these methods address only a small subset of Inpainting issues and these methods are not suitable for a wide variety of applications. 


\subsection{PDE based Inpainting.}

Partial Differential Equation (PDE) based algorithm is proposed by Bertalmio et.al [2]. This algorithm is the iterative algorithm. The main idea behind this algorithm is to continue geometric and photometric information that arrives at the border of the occluded area into area itself. This is done by propagating the information in the direction of minimal change using 'isophote lines'. This algorithm will produce good results if missed regions are small one. But when the missed regions are large this algorithm will take so long time and it will not produce good results. Then inspired by this work, Chan and Shen [3] proposed the Total Variational (TV) Inpainting model. This model uses Euler-Lagrange equation and anisotropic diffusion based on the strength of the isophotes. This model performs reasonably well for small regions and noise removal applications. But the drawback of this method is that this method neither connects broken edges nor greats texture patterns. The TV model then extended to CDD (Curvature Driven Diffusion) [4] model. In which it included the curvature information of the isophotes to handle the curved structures in a better manner. Then telea in [1] propose a fast marching method. This is considered as a PDE method which is faster and simpler to implement than other PDE based algorithms. All of the above mentioned algorithms are very time consuming and have some problems with the damaged regions with a large size. PDE based technique has been widely used in number of applications such as image segmentation, restoration etc. These algorithms were focused on maintaining the structure of the Inpainting area. And hence these algorithms produce blurred resulting image. Another drawback of these algorithms is that the large textured regions are not well reproduced.

\subsection{Exemplar based Inpainting.}

The exemplar based approach is an important class of inpainting algorithms. And they have proved to be very effective. Basically it consists of two basic steps: in the first step priority assignment is done and the second step consists of the selection of the best matching patch. The exemplar based approach samples the best matching patches from the known region, whose similarity is measured by certain metrics, and pastes into the target patches in the missing region.

Exemplar- based Inpainting iteratively synthesizes the unknown region i. e. target region, by the most similar patch in the source region. According to the filling order, the method fills structures in the missing regions using spatial information of neighbouring regions. This method is an efficient approach for reconstructing large target regions.

Generally, an exemplar-based Inpainting algorithm includes the following four main steps:

1) Initializing the Target Region, in which the initial missing areas are extracted and represented with appropriate data structures.

2) Computing Filling Priorities, in this a predefined priority function is used to compute the filling order for all unfilled pixels $p \in \delta \Omega$ in the beginning of each filling iteration.

3) Searching Example and Compositing, in which the most similar example is searched from the source region $\Phi$ to compose the given patch, $\Psi$ (of size $N \times N$ pixels) that centered on the given pixel p.

4) Updating Image Information, in which the boundary $\delta \Omega$ of the target region $\Omega$ and the required information for computing filling priorities are updated.

Numbers of algorithms are developed for the exemplar based image Inpainting. Such as, Jia [9] segmented an image into several regions based on its color texture features and then inpainted each region individually. Then Drori [10] proposed a fragment-based image Inpainting algorithm that iteratively approximated, searched, and added detail by compositing adaptive fragments. The computation time of this algorithm is intolerable. Bertalmio [11] developed a hybrid algorithm to combine the diffusion-based scheme [2] and texture synthesis [8]. This algorithm works well in recovering not only the geometrical structures but also the small texture regions. Then Criminisi [12] developed an efficient and simple approach to encourage fillingin from the boundary of the missing region where the strength of isophote nearby was strong, and then used the sum of squared difference (SSD) to select a best matching patch among the candidate source patches. In this algorithm of criminisi the region filling order is determined by the priority based mechanism. Cheng [13] generalized the priority function for the family of algorithms given in [12] to provide a more robust performance. Wong [14] developed a weighted similarity function. That function uses several source patches to reconstruct the target patch instead of using a single source patch. Wu [15] has proposed a cross isophotes exemplar-based model using the cross-isophote diffusion data and the local texture information which decided the dynamic size of exemplars. Komodakis [16] minimized a defined global objective function, which is efficient but computationally expensive. Sun [17] drew main curves manually to inpaint the missing structure first and then filled the unknown information using texture propagation. Hung [18] used the structure generation and Bezier curves to construct the missing edge information. Using the structure information and reconnecting contours by curve filling process, the damaged regions will be inpainted. Fang [20] developed a rapid image Inpainting system which consists of a multi-resolution training process and a patch-based image synthesis process. Xu [19] proposed two novel concepts of sparsity at the patch level for modeling the patch priority and patch representation. Compared with the diffusion-based approaches, the exemplar-based approaches achieve 
impressive results in recovering textures and repetitive structures no matter whether they are applied into the large regions or not.

Most of the new exemplar-based algorithms adopt the greedy strategy, so these algorithms suffer from the common problems of the greedy algorithm, being the filling order (namely priority) is very critical. Exemplar based Inpainting will produce good results only if the missing region consists of simple structure and texture. And if there are not enough samples in image then it is impossible to synthesize the desired image.

\subsection{Hybrid Inpainting.}

Hybrid inpainting technique is also called as Image Completion. It is used for filling large target (missing) regions. And also preserves both structure and texture in a visually plausible manner.The hybrid approaches combine both texture synthesis and PDE based Inpainting for completing the holes. The main idea behind these approaches is that it decomposed the image into two separate parts, Structure region and texture regions. The corresponding decomposed regions are filled by edge propagating algorithms and texture synthesis techniques. These algorithms are computationally intensive unless the fill region is small. One important direction we believe is more natural to the inpainting process is by structure completion through segmentation. This technique uses a two-step approach: the first stage is structure completion followed by texture synthesis. In the structure completion stage, segmentation, using the algorithm of, is performed based on the insouciant geometry, color and texture information on the input and then the partitioning boundaries are extrapolated to generate a complete segmentation for the input using tensor voting. The second step consists of synthesizing texture and color information in each segment, again using tensor voting.

\subsection{Semi-automatic and Fast Inpainting.}

Semi-automatic image inpainting requires user assistance. And it requires user assistance the in the form of guide lines to help in structure completion has found favour with researchers. The method by Jian et.al [19] proposed inpainting with Structure propagation. This technique follows a two-step process. In the first step a user manually specifies important missing information in the hole by sketching object boundaries from the known to the unknown region and then a patch based texture synthesis is used to generate the texture. The missing image patches are synthesized along the user specified curves by formulating the problem as a global optimization problem under various structural and consistency constraints. Simple dynamic programming can be used to derive the optimal answer if only a single curve is present. For multiple objects, the optimization is great deal more difficult and the proposes approximated the answer by using belief propagation. All the methods discussed above take minutes to hours to complete depending on the size of the Inpainting area and hence making it unacceptable for interactive user applications. To speed up the conventional image Inpainting algorithms, new classes of fast Inpainting techniques are being developed. Oliviera et.al [7] proposed a fast digital Inpainting technique based on an isotropic diffusion model which performs Inpainting by repeatedly convolving the Inpainting region with a diffusion kernel. A new method which treats the missing regions as level sets and uses Fast Marching Method (FMM) to propagate image information has been proposed by Telea in [1]. These fast techniques are not suitable in filling large hole regions as they lack explicit methods to inpaint edge regions. This technique results in blur effect in image.

\section{CONCLUSION}

In this paper we review the existing techniques of image Inpainting. We discussed a variety of image Inpainting techniques such as texture synthesis based Inpainting, PDE based Inpainting, Exemplar based Inpainting, Hybrid Inpainting, and semi-automatic and fast Inpainting techniques. For each technique we have provided a detailed explanation of the techniques which are used for filling the missing region making use of image. From this analysis, a number of shortcomings and limitations were highlighted of these techniques.

It is observed that the PDE based Inpainting algorithms cannot fill the large missing region and it cannot restore the texture pattern. The theoretical analysis proved that exemplar based Inpainting will produce good results for Inpainting the large missing region also these algorithms can inpaint both structure and textured image as well. But they work well only if missing region consists of simple structure and texture.

Further study includes development of efficient algorithm to reduce computational cost and to reduce the time required for Inpainting.

\section{REFERENCES}

[1] Telea,"An Image Inpainting Technique Based On The Fast Marching Method", Journal Of Graphics Tools, Vol.9, No. 1, ACM Press 2004.

[2] M. Bertalmio, G. Sapiro, V. Caselles, and C. Ballester, "Image inpainting," in Proceedings of SIGGRAPH, 2000, pp. 417-424.

[3] T. Chan and J. Shen, "Local inpainting models and TV inpainting," SIAM Journal on Applied Mathematics, Vol. 62, 2001, pp. 1019-1043.

[4] T. Chan and J. Shen, "Nontexture inpainting by curvature-driven diffusions," Journal of Visual Communication and Image Representation, Vol. 4, 2001, pp. 436-449. 
[5] D.J. Heeger And J.R. Bergen. Pyramid-Based Texture Analysis/Synthesis. In proceedings Of ACM Conf. Comp. Graphics (Siggraph), Volume 29, Pages $229\{233$, Los Angeles, Ca, 1995.

[6] C.W. Fang and J.J.J. Lien. Fast Image Replacement Using Multi-Resolution Approach. Jiaya Jia and Chi Keung Tang. Inference of Segmented Color and Texture Description by Tensor Voting. IEEE.

[7] M. Oliviera, B. Bowen, R. Mckenna, and Y.-S. Chang. Fast Digital Image Inpainting. In Proc. Of Intl. Conf. On Visualization, Imaging And Image Processing (VIIP), Page 261266, 2001.

[8] A. Efors and T. Leung, "Texture synthesis by non-parametric sampling," in Proceedings of the 17th IEEE International Conference on Computer Vision, 1999, pp. 1033-1038.

[9] J. Jia and C. K. Tang, "Image repairing: Robust image synthesis by adaptive nd tensor voting," in Proceedings of IEEE Computer Society Conference on Computer Vision Pattern Recognition, 2003, pp. 643-650.

[10] I. Drori, D. Cohen-Or, and H. Yeshurun, "Fragment-based image completion," ACM Transactions on Graphics, Vol. 22, 2003, pp. 303-312.

[11] M. Bertalmio, L. Vese, G. Sapiro, and S. Osher, "Simultaneous structure and texture image inpainting," IEEE Transactions on Image Processing, Vol. 12, 2003, pp. 882-889.

[12] A. Criminisi, P. Perez, and K. Toyama, "Region filling and object removal by exemplar- based image inpainting," IEEE Transactions on Image Processing, Vol. 13, 2004, pp. 1200-1212.

[13] W. Cheng, C. Hsieh, S. Lin, C. Wang, and J. Wu, "Robust algorithm for exemplar based image inpainting," in Proceedings of International Conference on Computer Graphics, Imaging and Visualization, 2005, pp. 64-69.

[14] A. Wong and J. Orchard, "A nonlocal means approach to exemplar-based inpainting," in Proceedings of the 15th IEEE International Conference on Image Processing, 2008, pp. 2600-2603.

[15] J. C. Huang, C. H. Hwang, Y. C. Liao, N. C. Tang, and T. J. Chen, "Exemplar-based image inpainting base on structure construction," Journal of Software, Vol. 3, 2008, pp. 57-64.

[16] N. Komodakis and G. Tziritas, "Image completion using efficient belief propagation via priority scheduling and dynamic pruning," IEEE Transactions on Image Processing, Vol. 16, 2007, pp. 2649-2661.

[17] J. Sun, L. Yuan, J. Jia, and H. Y. Shum, "Image completion with structure propagation," in Proceedings of SIGGRAPH, 2005, pp. 861-868.

[18] J. Y. Wu and Q. Q. Ruan, “A novel exemplar-based image completion model," Journal of Information Science and Engineering, Vol. 25, 2009, pp. 481-497.

[19] Z. Xu and S. Jian, "Image inpainting by patch propagation using patch sparsity," IEEE Transactions on Image Processing, Vol. 19,2010, pp. $1153-1165$

[20] C. Fang and J. J. Lien, "Rapid image completion system using multiresolution patch based directional and nondirectional approaches," IEEE Transactions on Image Processing, Vol. 18, 2009, pp. 2769-2779. 